\title{
A RELAÇÃO EDUCAÇÃO E SAÚDE: UMA ABORDAGEM SOBRE O EXCESSO DE CONSUMO DE AÇÚCAR PELO JOVEM
}

\author{
Viviane Terezinha Sebalhos Dalmolin ${ }^{1}$, Paulo Edelvar Corrêa Peres², \\ Jorge Orlando Cuellar Noguera ${ }^{3}$ \\ ${ }^{1}$ Universidade Federal de Santa Maria, vidalmolin@gmail.com \\ 2 Universidade Federal de Santa Maria, pecperes@yahoo.com.br \\ ${ }^{3}$ Universidade Federal de Santa Maria, jocunoguera@hotmail.com
}

http://dx.doi.org/10.5902/223613086577

\section{RESUMO}

Atualmente há um grande consumo de alimentos com excesso de açúcar pelo jovem, devido principalmente a sua disponibilidade no mercado e aos interesses econômicos e culturais do meio em que vive. Torna-se necessária, portanto, a intervenção na educação alimentar, quer da família, quer da escola ou grupos de formação, pois este grupo é alvo fácil da mídia e dos fortes interesses econômicos, onde o menos importante é a saúde dos consumidores. O objetivo principal deste trabalho foi avaliar a eficiência no aprendizado nutricional dos jovens através de atividades extraclasse (diálogo-palestra), com profissionais da área da saúde. Foram obtidas informações sobre o conhecimento e hábitos alimentares de jovens da oitava série do ensino fundamental $\left(9^{\circ}\right.$ ano $)$ e da primeira série do ensino médio, oriundos de escola pública e particular da rede de ensino de Santa Maria, RS. O tema foi abordado a partir de questionários investigativos, antes $e$ após as ações de conscientização, denominados pré-teste e pós-teste, respectivamente. Após as ações de sensibilização, o nível de conscientização aumentou entre o grupo pesquisado, havendo o claro indicativo de intenção da redução no consumo de açúcar e de produtos açucarados. A escola, como veículo de aprendizagem, mostra-se um local apropriado para debater as questões nutricionais e de saúde proporcionando uma educação sustentável, de base forte e de caráter multiplicadora.

Palavras-chave: açúcar, cárie, obesidade, ação educacional.

\begin{abstract}
Currently there has been a large consumption of foods with excess sugar by teenagers, mainly due to its availability in the market and the economic and cultural interests of the environment in which they live in. It becomes necessary, therefore, to intervene in food education, through family, school or training groups, because this demographic group is an easy target for the media and strong economic interests, to whom the health of consumers is of the least importance. The main objective of this study was to evaluate the efficiency of extra-class activities (lecturedialogue), with health professionals in teaching teenagers about nutrition. We obtained information on the knowledge and eating habits of students from eighth grade (9th grade) and the first grade of high school (secondary schools), from both the public and private school system in Santa Maria, RS. The subject was approached through investigative questionnaires before and after the awareness actions, called pre-test and post-test respectively. After the lecturesdialogues, awareness levels increased among the studied group and there was a clear indication of the intention of reducing the consumption of sugar and sugary products. The school as a learning
\end{abstract}


vehicle appears to be an appropriate place to discuss nutrition and health education issuesby providing a sustainable education of strong base and multipliercharacter.

Key Words: Sugar, dental decay, obesity, educational action.

\section{INTRODUÇÃO}

Em geral, é alarmante a falta de bons hábitos alimentares especialmente pelo consumo abusivo e muitas vezes inconsciente de açúcar (sacarose). Principalmente os jovens, são submetidos cada vez mais a um hiperconsumo calórico e ao sedentarismo, pois encontram-se como protagonistas para a indústria de alimentos, das cadeias de "fast food" e das propagandas de TV. (SICHIERI \& SOUZA ,2008).

Para mantermos o metabolismo energético corporal é necessária a ingestão de alimentos, onde o nível basal de glicose no sangue, em torno de 80 - $90 \mathrm{mg} \mathrm{dL}^{-1}$, passa para $130-140 \mathrm{mg} \mathrm{dL}^{-}$ ${ }^{1}$ quando a glicose chega ao sangue. Paralelamente, o nível de insulina aumenta, propiciando a passagem da glicose para o interior celular, onde qualquer variação causará transtornos, sendo que o estímulo mais potente à produção de insulina é o provocado pela glicose que provém do açúcar refinado, ativando fortemente o pâncreas pelo consumo excessivo por meses e anos seguidos (FUKUDA, 2004; BESSESEN, 2001).

De acordo com Bessesen (2001), o metabolismo acelerado produz o estado denominado resistência à insulina, onde a célula, em um mecanismo de defesa, passa a rejeitar o excesso de glicose, onde, mesmo havendo grande quantidade de insulina na corrente sanguínea, diminuirá a passagem da glicose para o interior da célula, caracterizando situação de hiperinsulinemia e hiperglicemia.

Em tempos passados, as doenças infecciosas lideravam as causas de mortes. Com os avanços tecnológicos e controle das infecções, essas causas foram substituídas pela alta incidência das doenças crônicas não transmissíveis, tais como: obesidade, hipertensão arterial, doenças coronarianas, diabetes e suas implicações, grande parte delas relacionadas aos hábitos alimentares, especialmente ao excesso de açúcar e gorduras (LESSA, 1998). Uma das doenças crônicas, segundo Sartorelli \& Franco (2003), é a prevalência do diabetes mellitus tipo 2, especialmente em jovens. O índice dessa doença crônica tem se elevado devido ao aumento das taxas de obesidade associada à vida sedentária e as modificações no consumo alimentar.

O aumento da obesidade é nitidamente multifatorial, sendo que o aumento de sua incidência nos últimos anos tem sido explicado por sociólogos e nutrólogos relacionando fatores nutricionais inadequados (excessivo consumo de lanches, guloseimas, etc) e um excessivo sedentarismo (TV, vídeo-games, etc), relacionados com base bioquímica e hormonal controlados por neurotransmissores, que interferem no comportamento alimentar (DAMIANI, 2000).

Em relação à cárie, a sacarose foi fortemente identificada como o principal açúcar implicado em sua etiologia. A prevalência e severidade desta doença têm aumentado rapidamente nos países em desenvolvimento, tendo como um fator relevante à disponibilidade dos açúcares. (FREIRE et al.,1994).

Para Caroba \& Silva (2005), a fase da adolescência é o momento privilegiado para as intervenções no ramo nutricional, buscando a adoção de hábitos alimentares saudáveis, visto que nessa fase, os processos de crescimento e maturação, tanto do ponto de vista somático como psicológico estão em desenvolvimento. 
Na área da saúde, a interdisciplinaridade precisa contribuir a fim de reorientar os efeitos da fragmentação do conhecimento, valorizando sua complexidade, que respeita as bases disciplinares específicas, mas busca soluções compartilhadas, possibilitando ações em conjunto e dessa forma, investindo em estratégias para a realização de ações eficazes (SAUPE et al., 2005).

A Educação Ambiental encontra-se inserida na era globalizada, portanto na abordagem da educação nutricional proporciona um processo de modificação, com a participação dos indivíduos na identificação dos problemas, assim como na busca de soluções. É reconhecida sua eficácia pela constituição brasileira, que declara que todo o brasileiro tem o direito à educação ambiental e o Estado tem o dever de promovê-la em todos os níveis de ensino (CASSOL, 2012).

Para Zan et al (2012), a vivência do cotidiano com aplicações de propostas de caráter prático e a educação ambiental direcionadas ao ensino interdisciplinar, poderá levar a situações que estimulem aprendizagem resultando em uma sociedade sustentável e consciente.

Dessa forma, esse trabalho tem como objetivo avaliar a eficiência no aprendizado nutricional dos jovens através de atividades extraclasse (diálogo-palestra), sensibilizando-os sobre a importância dos bons hábitos alimentares, provocando, portanto, uma reflexão sobre os possíveis danos causados pelo excesso de ingestão de açúcar (sacarose).

\section{MATERIAL E MÉTODOS}

Foram obtidas informações sobre o conhecimento e hábitos alimentares de jovens da oitava série do ensino fundamental $\left(9^{\circ}\right.$ ano) e da primeira série do ensino médio, oriundos de escola pública e particular da rede de ensino de Santa Maria, no estado do Rio Grande do Sul, pertencente a 8a Coordenadoria Regional de Educação.

O tema foi abordado a partir de questionários investigativos, antes das ações de conscientização, denominado pré-teste, com a participação de 148 jovens e após as ações, denominado pós-teste, com a participação de 136 jovens. O número de jovens participantes é de ordem casual pertencentes às séries envolvidas no projeto

As ações de conscientização constaram de palestras proferidas ao público dessa pesquisa, com profissionais da área de saúde (nutricionistas e odontólogos), abordando as conseqüências metabólicas, obesidade, diabetes e cárie pelo excesso de consumo de açúcar.

Para comparar os resultados do pré-teste e pós-teste, fazendo relação com os resultados obtidos antes e após as ações de conscientização, foi realizada a análise estatística, teste do Qui quadrado, utilizando o Software Statistica 7 (StatSoft, 2004). Este teste mediu a probabilidade das diferenças encontradas nos grupos de amostras, verificando se a freqüência absoluta observada de uma variável é significativamente diferente da distribuição de freqüência absoluta esperada. Dessa forma, o princípio básico deste procedimento estatístico foi comparar as possíveis diferenças dos resultados entre ensino público e privado, ensino fundamental e médio na freqüência e qualidade de consumo e também no grau de informações sobre o tema estudado, através de dados de contagem.

Foi utilizada a relação:

$$
X^{2}=\Sigma\left[(0-e)^{2} / e\right]
$$

onde:

$X^{2}=$ teste do qui quadrado-probabilidade de ocorrência.

$\Sigma=$ somatório

$0=$ freqüência observada

e = freqüência esperada 
Se a probabilidade for alta não há diferenças estatisticamente significativas. Se a probabilidade for baixa (menor que $5 \%$ ) há diferenças estatisticamente significativas.

\section{RESULTADOS E DISCUSSÃO}

1- pré-teste

Conforme os dados apresentados na tabela 1, não existem diferenças no consumo diário de açúcar entre os jovens que freqüentam a escola pública e a particular, assim como não existem diferenças entre a escolaridade dos pesquisados, igualando-se o consumo tanto no ensino fundamental como no ensino médio (Tabela 2). Essas observações vão de acordo com Brasil (2007), que relata que o consumo de açúcar é elevado e vem aumentado nos últimos anos, tornando o Brasil um dos maiores consumidores deste produto.

Tabela 1 - Freqüências observadas e esperadas (entre parênteses) do consumo diário de açúcar dos estudantes da rede de pública e particular.

\begin{tabular}{cccc}
\hline \multirow{2}{*}{ Consumo diário } & \multicolumn{3}{c}{ Escola } \\
\cline { 2 - 4 } & Pública & Particular & Total \\
\hline \hline Não consome & $3(3,44)$ & $2(1,55)$ & $\mathbf{5}$ \\
1 vez ao dia & $7(11,71)$ & $10(5,28)$ & $\mathbf{1 7}$ \\
2 vezes ao dia & $26(25,5)$ & $11(11,5)$ & $\mathbf{3 7}$ \\
3 vezes ao dia & $24(24,12)$ & $11(10,87)$ & $\mathbf{3 5}$ \\
$>$ de 3 vezes ao dia & $42(37,21)$ & $12(16,78)$ & $\mathbf{5 4}$ \\
\hline Total & $\mathbf{1 0 2}$ & $\mathbf{4 6}$ & $\mathbf{1 4 8}$
\end{tabular}

teste do $Q$-quadrado $=8,30(4 \mathrm{GL}$ ) $\operatorname{com} p=0,08$ (maior que 0,05 )

Tabela 2 - Freqüências observadas e esperadas (entre parênteses) do consumo diário de açúcar dos estudantes do ensino fundamental e médio.

\begin{tabular}{cccc}
\hline \multirow{2}{*}{ Consumo diário } & \multicolumn{3}{c}{ Escolaridade } \\
\cline { 2 - 4 } & Ensino fundamental & Ensino médio & Total \\
\hline \hline Não consome & $4(2,40)$ & $0(1,59)$ & $\mathbf{4}$ \\
1 vez ao dia & $12(10,82)$ & $6(7,17)$ & $\mathbf{1 8}$ \\
2 vezes ao dia & $19(22,25)$ & $18(14,75)$ & $\mathbf{3 7}$ \\
3 vezes ao dia & $21(21,04)$ & $14(13,95)$ & $\mathbf{3 5}$ \\
$>$ de 3 vezes ao dia & $33(32,47)$ & $21(21,52)$ & $\mathbf{5 4}$ \\
\hline Total & $\mathbf{8 9}$ & $\mathbf{5 9}$ & $\mathbf{1 4 8}$ \\
\hline
\end{tabular}

teste do $Q$-quadrado $=4,18(4 \mathrm{GL}) \operatorname{com} p=0,38$ (maior que 0,05 )

Questionados sobre os locais de maior consumo de açúcar, foi constatado que não há diferença nas respostas entre as escolas públicas e particulares e entre ensino fundamental e médio, sendo que o local mais freqüente de consumo para todo o grupo foi no seu próprio domicílio, seguido das festas e recreios das escolas, respectivamente. (Tabela 3 e 4). Conforme 
Damiani (2000), os interferentes no comportamento alimentar estão relacionados a fatores nutricionais inadequados (excessivo consumo de lanches, guloseimas, etc.), excessivo sedentarismo (TV, vídeos-game, etc.), e base bioquímica e hormonal, onde estão diretamente associados aos hábitos familiares e adquiridos na convivência social e escolar.

Tabela 3 - Freqüências observadas e esperadas (entre parênteses) relacionadas ao local de consumo de açúcar e a rede de ensino.

\begin{tabular}{cccc}
\hline \multirow{2}{*}{ Local de consumo } & \multicolumn{3}{c}{ Escola } \\
\cline { 2 - 4 } & Pública & Particular & Total \\
\hline \hline em casa & $77(75,63)$ & $29(30,36)$ & $\mathbf{1 0 6}$ \\
na escola, sala de aula & $14(16,41)$ & $4(6,58)$ & $\mathbf{2 3}$ \\
na escola, recreio & $21(21,40)$ & $9(8,59)$ & $\mathbf{3 0}$ \\
no clube & $2(1,42)$ & $1(0,57)$ & $\mathbf{2}$ \\
Festas & $23(17,12)$ & $12(6,87)$ & $\mathbf{2 4}$ \\
\hline Total & $\mathbf{1 3 7}$ & $\mathbf{5 5}$ & $\mathbf{1 9 2}$ \\
\hline
\end{tabular}

teste do $Q$-quadrado $=1,05(4 \mathrm{GL}) \operatorname{com} p=0,90$ (maior que 0,05 )

Tabela 4 - Freqüências observadas e esperadas (entre parênteses) relacionadas ao local de consumo de açúcar e o nível de escolaridade.

\begin{tabular}{cccc}
\hline & \multicolumn{3}{c}{ Escolaridade } \\
\cline { 2 - 4 } Local de consumo & Fundamental & Médio & Total \\
\hline \hline em casa & $60(64,15)$ & $46(41,84)$ & 106 \\
na escola, sala de aula & $16(10,89)$ & $4(7,10)$ & 18 \\
na escola, recreio & $19(18,15)$ & $11(11,84)$ & 30 \\
no clube & $1(0,60)$ & $0(0,39)$ & 1 \\
Festas & $20(21,18)$ & $15(13,81)$ & 35 \\
\hline Total & $\mathbf{1 1 6}$ & $\mathbf{7 6}$ & $\mathbf{1 9 2}$ \\
\hline
\end{tabular}

teste do $Q$-quadrado $=5,52(4 \mathrm{GL}) \operatorname{com} p=0,24$ (maior que 0,05 )

Ao serem questionados sobre as principais doenças observadas em seus familiares (Tabelas 5 e 6), foi observado que, independente do tipo de escola ou do grau de escolaridade, a doença mais freqüente nas famílias dos jovens é diabetes, concordando com Sartorelli \& Franco (2003), que relata que o índice dessa doença crônica tem se elevado devido ao aumento das taxas de obesidade associada à vida sedentária e as modificações no consumo alimentar. 
Tabela 5 - Freqüências observadas e esperadas (entre parênteses) relacionadas as principais doenças na família e rede escolar.

\begin{tabular}{cccc}
\hline \multirow{2}{*}{ Problemas de saúde } & \multicolumn{2}{c}{ Escola } & \multirow{2}{*}{ Total } \\
\cline { 2 - 3 } & Pública & Particular & $\mathbf{3 7}$ \\
\hline \hline Obesidade & $28(26,42)$ & $9(10,57)$ & $\mathbf{6 2}$ \\
cárie dentária & $46(44,28)$ & $16(17,71)$ & $\mathbf{7 7}$ \\
Diabetes & $55(55)$ & $22(22)$ & $\mathbf{4 7}$ \\
Labirintite & $33(33,57)$ & $14(13,43)$ & $\mathbf{4 1}$ \\
problemas coronários & $26(29,28)$ & $15(11,71)$ & $\mathbf{2 3}$ \\
problemas vasculares & $17(16,42)$ & $6(6,57)$ & $\mathbf{2 8 7}$ \\
\hline Total & $\mathbf{2 0 5}$ & $\mathbf{8 2}$ &
\end{tabular}

teste do $Q$-quadrado $=1,95(5 \mathrm{GL}$ ) com $p=0,86$ (maior que 0,05 )

Tabela 6 - Freqüências observadas e esperadas (entre parênteses) relacionadas as principais doenças na família e o nível de escolaridade.

\begin{tabular}{|c|c|c|c|}
\hline \multirow{2}{*}{ Problemas de saúde } & \multicolumn{2}{|c|}{ Escolaridade } & \multirow{2}{*}{ Total } \\
\hline & Fundamental & Médio & \\
\hline Obesidade & $21(22,52)$ & $16(14,47)$ & 37 \\
\hline cárie dentária & $35(37,74)$ & $27(24,25)$ & 62 \\
\hline Diabetes & $51(49,31)$ & $30(31,68)$ & 81 \\
\hline Labirintite & $16(17,65)$ & $13(11,34)$ & 29 \\
\hline problemas coronários & $25(23,13)$ & $13(14,86)$ & 38 \\
\hline problemas vasculares & $17(14,61)$ & $7(9,38)$ & 24 \\
\hline Total & 165 & 106 & 271 \\
\hline
\end{tabular}

teste do $Q$-quadrado $=2,70(5 \mathrm{GL}) \operatorname{com} p=0,75$ (maior que 0,05 )

2- pós-teste

Ao serem questionados sobre como seria o consumo de açúcar após as palestras, observou-se a eficácia das mesmas, pois os jovens responderam que a intenção deles é diminuir a quantidade de alimentos açucarados (Tabelas 7 e 8). De acordo com Caroba \& Silva (2005), a fase da adolescência é o momento privilegiado para as intervenções no ramo nutricional, pois nessa fase, os processos de crescimento e maturação, tanto do ponto de vista somático como psicológico estão em desenvolvimento. 
Tabela 7- Freqüências observadas e esperadas (entre parênteses), na escola da rede pública e privada com relação ao consumo de açúcar após as palestras.

\begin{tabular}{cccc}
\hline \multirow{2}{*}{ Consumo de açúcar } & \multicolumn{3}{c}{ Escola } \\
\cline { 2 - 4 } & Pública & Particular & Total \\
\hline \hline aumentar & $1(2,75)$ & $4(2,24)$ & $\mathbf{5}$ \\
diminuir & $53(53,49)$ & $44(43,50)$ & $\mathbf{9 7}$ \\
manter-se & $21(18,75)$ & $13(15,25)$ & $\mathbf{3 4}$ \\
\hline Total & $\mathbf{7 5}$ & $\mathbf{6 1}$ & $\mathbf{1 3 6}$ \\
\hline
\end{tabular}

teste do $Q$-quadrado $=3,10(2 \mathrm{GL}) \operatorname{com} p=0,21$ (maior que 0,05 )

Tabela 8 - Freqüências observadas e esperadas (entre parênteses), na escola de nível fundamental e médio com relação ao consumo de açúcar após as palestras.

\begin{tabular}{cccc}
\hline \multirow{2}{*}{ Consumo de açúcar } & \multicolumn{3}{c}{ Nível de escolaridade } \\
\cline { 2 - 4 } & Fundamental & Médio & Total \\
\hline \hline Aumentar & 3 & 2 & $\mathbf{5}$ \\
Diminuir & 49 & 48 & $\mathbf{9 7}$ \\
manter-se & 23 & 11 & $\mathbf{3 4}$ \\
\hline Total & $\mathbf{7 5}$ & $\mathbf{6 1}$ & $\mathbf{1 3 6}$ \\
\hline
\end{tabular}

teste do $Q$-quadrado $=3,03(2 \mathrm{GL}) \operatorname{com} p=0,22$ (maior que 0,05 )

Os resultados obtidos nessa pesquisa indicam que os jovens independentes do tipo de escola e do nível de escolaridade perceberam após as palestras que houve sensibilização sobre a informação do grande número de alimentos açucarados descritos nas palestras. A percepção dos jovens aumentou e eles tomaram consciência de que quase todos os alimentos possuem açúcar, inclusive os salgados. Carvalho (2006) faz um amplo relato sobre a incidência elevada de açúcar nos alimentos, especialmente os industrializados.

Objetivando avaliar a importância do conhecimento sobre o tema alimentação e saúde relacionando os resultados no pré-teste com o pós-teste, obtiveram-se os resultados descritos na tabela 9.

Tabela 9 - Freqüências observadas e esperadas (entre parênteses) do tema alimentação e saúde no pré-teste e pós-teste.

\begin{tabular}{cccc}
\hline \multirow{2}{*}{ Conhecimento } & \multicolumn{3}{c}{ Teste } \\
\cline { 2 - 4 } & Pré teste & Pós teste & Total \\
\hline \hline $\operatorname{Sim}$ & $142(143,22)$ & $128(126,77)$ & $\mathbf{2 7 0}$ \\
Não & $6(4,77)$ & $3(4,22)$ & $\mathbf{9}$ \\
\hline Total & $\mathbf{1 4 8}$ & $\mathbf{1 3 1}$ & $\mathbf{2 7 9}$ \\
\hline
\end{tabular}

teste do $Q$-quadrado $=0,69(1 \mathrm{GL}) \operatorname{com} p=0,941$ (maior que 0,05 ).

Não existe relação entre o tema e o pré-teste e pós teste, observando que os alunos das diferentes escolas e diferentes graus de escolaridade consideram importante o conhecimento sobre alimentação e saúde, o que permaneceu antes e após as palestras ministradas, confirmando 
que a adolescência é o momento mais apropriado para inferir comportamentos até então não adquiridos na convivência familiar através de intervenções pela área da saúde e nutrição, na busca da adoção de bons hábitos alimentares e promoção da saúde na vida adulta (Caroba \& Silva, 2005).

\section{CONCLUSÃO}

O público jovem pesquisado consome diariamente quantidade excessiva de açúcar ou produtos açucarados. Esse consumo ocorre mesmo que os jovens façam a relação com algumas doenças como diabetes e cárie, sendo o seu domicílio o local onde o consumo é maior, indicando que a influência da família nos hábitos alimentares dos jovens é de grande importância na educação nutricional.

As ações de sensibilização aumentou o nível de conscientização do grupo pesquisado, havendo o claro indicativo de intenção da redução no consumo de açúcar e de produtos açucarados mostrando que é possível relacionar educação e saúde de forma a minimizar problemas futuros no âmbito da saúde,contribuindo para um futuro com menores índices de casos de cárie dentária, obesidade, diabetes, entre outros.

A iniciativa de levar à escola a possibilidade de juntos, educação e saúde, proporcionar o contínuo e crescente ideal de uma educação sustentável, de base forte e de caráter multiplicadora foi demonstrada nesse trabalho.

\section{BIBLIOGRAFIA}

BESSESEN, D.H. The role of carbohydrates in insuline resistence. Journal of Nutrition. v. 131, n. 10, p. 2782-2786, 2001.

BRASIL. Ministério da Agricultura, Pecuária e Abastecimento. Balanço nacional da cana-de-açúcar e agroenergia. Brasília, DF, 2007. 140 p.

CAROBA, D.C.R; SILVA, M. V. Consumo alimentar de adolescentes matriculados na rede pública de ensino de Piracicaba- SP. Revista de Segurança alimentar e Nutricional. v. 12, n. 1, p. 55-66, 2005.

CARVALHO, F.A.C. O livro negro do açúcar - Algumas verdades sobre a indústria do açúcar, Rio de Janeiro (RJ) 2006, 205 p. Disponível em:

http://www.sunnet.com.br/textos/O_Livro_negro_do_acucar.pdf Acesso em: 21/08/12.

CASSOL, P.B. O verde abalou o muro. Revista Eletrônica em Gestão, Educação e Tecnologia Ambiental. Vol. 5, No 5. p.767-772 (2012). Disponível em:

http://cascavel.ufsm.br/revistas/ojs-2.2.2/index.php/reget/article/viewFile/4211/2805 Acesso em: 20/08/12.

DAMIANI, D. Obesidade na Infância e Adolescência - Um Extraordinário Desafio! Arquivos Brasileiro de Endocrinologia Metabologia. v.44, n.5, p. 363-365, 2000. 
FREIRE, M.C.M., CANNON, G., SHEIHAM, B. Análise das recomendações internacionais sobre o consumo de açúcares publicadas entre 1961 a 1991. Revista de saúde Pública. v.28 n.3 p. :228237, 1994

FUKUDA, Y. Açúcar: Amigo ou Vilão? Barueri (SP): Manole, 2004, 161p.

LESSA I. O adulto brasileiro e as doenças da modernidade: epidemiologia das doenças crônicas não transmissíveis. São Paulo: Editora Hucitec/Abrasco. 1998.

SARTORELLI, D.S.; FRANCO, L.J. Tendências do diabetes mellitus no Brasil: o papel da transição nutricional. Caderno de Saúde Pública v.19 sup 1, p.29-36, 2003.

SAUPE, R.; CUTOLO, L.R.A.; WENDHAUSEN, A.L.P.; BENITO, G.A.V.;Competência dos profissionais da saúde para o trabalho interdisciplinar. Interface, Comunicação, Saúde, Educação. V.9, n. 18, p.521-536, 2005.

SICHIERI R. E SOUZA R. A. Estratégias para prevenção da obesidade em crianças e adolescentes Caderno de Saúde Pública, 24sup, p.209-234, 2008.

STATSOFT, Inc. STATISTICA (data analysis software system), version 7. 2004.

ZAN et al. Ensino interdisciplinar da educação ambiental nas disciplinas de biologia e química do ensino médio: uma proposta para as escolas públicas do município de ariquemes, Rondônia, Brasil. Revista "Monografias Ambientais - REMOA/UFSM. v(7), no 7, p. 1630 - 1645, MAR-JUN, 2012. Disponível em: http://cascavel.ufsm.br/revistas/ojs-

2.2.2/index.php/remoa/article/view/5226/3307 Acesso em: 10/09/12 